\title{
Progression of Aortic Calcification in Stage 4-5 Chronic Kidney Disease Patients Transitioning to Dialysis and Transplantation
}

\author{
Roosa Lankinen ${ }^{a}$ Markus Hakamäki ${ }^{a}$ Tapio Hellmana Niina S. Koivuviita ${ }^{a}$ \\ Kaj Metsärinne ${ }^{a} \quad$ Mikko J. Järvisalo ${ }^{a, b, c}$ \\ aKidney Centre, Turku University Hospital and University of Turku, Turku, Finland; 'bepartment of Anaesthesiology \\ and Intensive Care, University of Turku, Turku, Finland; CPerioperative Services, Intensive Care and Pain Medicine, \\ Turku University Hospital, Turku, Finland
}

\section{Keywords}

Cardiovascular disease · Chronic kidney disease · Aortic calcification

\begin{abstract}
Background and Aims: Abdominal aortic calcification (AAC) is common in chronic kidney disease (CKD) patients and associated with increased mortality. Comparative data on the AAC score progression in CKD patients transitioning from conservative treatment to different modalities of renal replacement therapy (RRT) are lacking and were examined. Methods: 150 study patients underwent lateral lumbar radiograph to study AAC in the beginning of the study before commencing RRT (AAC1) and at 3 years of follow-up (AAC2). We examined the associations between repeated laboratory tests taken every 3 months, echocardiographic and clinical variables and $A A C$ increment per year $(\triangle A A C)$, and the association between $\triangle A A C$ and outcomes during follow-up. $\boldsymbol{R e}$ sults: At the time of AAC2 measurement, 39 patients were on hemodialysis, 39 on peritoneal dialysis, 39 had a transplant, and 33 were on conservative treatment. Median AAC1 was $4.8(0.5-9.0)$ and median AAC2 $8.0(1.5-12.0)(p<0.0001)$. $\triangle \mathrm{AAC}$ was similar across the treatment groups $(p=0.19)$.
\end{abstract}

$\triangle \mathrm{AAC}$ was independently associated with mean left ventricular mass index (LVMI) (log LVMI: $\beta=0.97, p=0.02$ ) and mean phosphorus through follow-up (log phosphorus: $\beta=1.19$, $p=0.02$ ) in the multivariable model. Time to transplantation was associated with $\triangle \mathrm{A} A C$ in transplant recipients (per month on the waiting list: $\beta=0.04, p=0.001$ ). $\triangle A A C$ was associated with mortality (HR 1.427, 95\% confidence interval 1.044-1.950, $p=0.03$ ). Conclusion: AAC progresses rapidly in patients with $C K D$, and $\triangle A A C$ is similar across the CKD treatment groups including transplant recipients. The increment rate is associated with mortality and in transplant recipients with the time on the transplant waiting list.

\footnotetext{
(c) 2021 The Author(s).

Published by S. Karger AG, Basel
}

\section{Introduction}

Patients with chronic kidney disease (CKD) are at remarkably high risk for cardiovascular events and death [1]. Atherosclerosis and arterial calcification often progress rapidly in CKD patients, a phenomenon which is inadequately explained by traditional risk factors such as diabetes, smoking, obesity, dyslipidemia, and hypertension [2]. CKD-mineral and bone disorder (CKD-MBD)
(C) 2021 The Author(s)

Published by S. Karger AG, Basel

This is an Open Access article licensed under the Creative Commons Attribution-NonCommercial-4.0 International License (CC BY-NC) (http://www.karger.com/Services/OpenAccessLicense), applicable to the online version of the article only. Usage and distribution for commercial purposes requires written permission.
Correspondence to:

Roosa Lankinen, roosa.lankinen@ @tyks.fi 
syndrome leads to arterial calcification, which is further augmented by progression of CKD [3]. In end-stage kidney disease, vascular calcification (VC) is characterized by mineral deposition in the tunica media of the arterial wall. Previous data suggest that the pathophysiological mechanism of $\mathrm{VC}$ is a dynamic process, rather than passive mineral precipitation [4].

The abdominal aortic calcification (AAC) score is independently associated with cardiovascular events and death in the general population and in dialysis patients [5-7], and thus current guidelines recommend the evaluation of AAC in CKD [3]. Furthermore, previous studies in moderate CKD, maintenance dialysis, and kidney transplant patients have shown that VC is common and progresses even following successful kidney transplantation $[8,9]$. In fact, Noordzij and coworkers demonstrated progression of aortic calcification in almost a third of 384 patients on maintenance hemodialysis (HD) or peritoneal dialysis (PD) during a mean follow-up of 2.3 years, and progressed calcification was significantly associated with mortality [10]. Some previous studies have linked accelerated progression of VC with hypercalcemia and hyperparathyreosis in maintenance dialysis patients $[10$, 11]. Most previous prospective studies have however examined the progression of AAC in patients already on maintenance dialysis or in patients with prior kidney transplantation, whereas data on AAC progression in CKD stage 4-5 patients transitioning from conservative treatment to different modalities of renal replacement therapy (RRT) are lacking.

Therefore, we aimed to study progression of AAC, associated risk factors, and outcomes in CKD stage 4-5 patients transitioning to dialysis and kidney transplantation. Furthermore, the aim of the study was to compare AAC progression between different modalities of RRT.

\section{Materials and Methods}

\section{Study Protocol}

Two hundred ten consecutive patients referred to the Kidney Centre Predialysis Outpatient Clinic of Turku University Hospital were recruited between August 2013 and September 2017 in the Chronic Arterial Disease, quality of life and mortality in chronic KIDney injury (CADKID) study. The study population target was set to a minimum of 200 patients in the beginning of the recruitment. CADKID is an ongoing, prospective, follow-up study assessing arterial disease, quality of life, mortality, and their predictors in patients with CKD (CKD-KDIGO 4-5) (http://www.ClinicalTrials.gov NCT04223726) [12]. CKD stage 4-5 patients are followed up regularly at 1 - to 2 -month intervals at the Kidney Centre Predialysis Outpatient Clinic. The individual patient data were collected from the research hospital's patient documents and during study and clinical control visits. The data from hospital software were combined and the patient identity numbers removed before the statistical analyses.

The present study is a prespecified report from the CADKID study aiming to examine the progression of calcification and associated risk factors including the treatment modality for CKD. At least one AAC measurement was available in 199 patients, and 150 patients had 2 consecutive AAC measurements and comprised the study cohort. The CADKID study baseline imaging data including baseline AAC measurements have been previously published [12]. Laboratory variables including blood hemoglobin, leukocytes, thrombocytes, glycated hemoglobin, $\mathrm{pH}$, bicarbonate and base excess, plasma C-reactive protein, glucose, alanine aminotransferase, alkaline phosphatase, creatinine, urea, albumin, sodium, potassium, phosphorus, total and ionized calcium and parathyroid hormone (PTH), and serum lipids were collected and recorded every 3 months from the beginning of the study spanning to the second AAC imaging in every patient. The estimated glomerular filtration rate (eGFR) was calculated using the Chronic Kidney Disease Epidemiology Collaboration equation. Major adverse cardiovascular events (MACEs) defined as a composite of cardiovascular death, myocardial infarction, stroke, and coronary artery revascularization were recorded.

\section{Assessment of $A A C$}

Lateral lumbar radiography was performed in the standing position using standard radiographic equipment in the beginning of the study and at 3 years of follow-up. At our center, the measurement of AAC from lateral lumbar radiographs is part of standard clinical assessment in CKD patients when considering their eligibility to the transplant waiting list. Calcification of the abdominal aorta was graded using a previously validated system in the region corresponding to the first through the fourth lumbar vertebrae [13]. Both the location and the severity of calcific deposits at each lumbar vertebral segment were evaluated. Lesions were graded as follows: 0 , no aortic calcific deposits; 1 , small scattered calcific deposits filling $<1 / 3$ of the longitudinal wall of the aorta; 2 , calcific lesions filling $1 / 3$ or more but less than $2 / 3$ of the longitudinal aortic wall; and 3, two-thirds or more of the longitudinal wall of the aorta calcified (online suppl. Fig. 1; for all online suppl. material, see www.karger.com/doi/10.1159/000518670). In the anteroposterior severity score $(0-24)$, the scores of individual aortic segments both for the posterior and anterior wall were summed. All radiographs were read independently by 2 researchers, and the mean AAC score was used in the analyses. The intra- and interobserver variations (CVs) of AAC measurements were $4.8 \%$ and $11.5 \%$, and the mean differences between measurements 0.5 and 1.0 points, respectively. AAC increment per year $(\triangle \mathrm{AAC})$ was defined as: (follow-up AAC [AAC2] - baseline AAC [AAC1])/time between $\mathrm{AAC} 1$ and $\mathrm{AAC} 2$ assessments (years).

\section{Echocardiography}

The echocardiographic measures were collected from standardized transthoracic echocardiography performed in the beginning of the study and repeated at 3 years of follow-up (available in 143 patients). The systolic and diastolic dimensions and function of the left ventricle as well as left ventricular wall thicknesses were measured. The left ventricular mass index (LVMI) was calculated using the following equations: LVMI = left ventricular mass/body 
Table 1. Demographic, clinical, laboratory, and echocardiographic characteristics

\begin{tabular}{|c|c|}
\hline Variable & Value \\
\hline Number of subjects, female, $n(\%)$ & $150(52)$ \\
\hline Age, years & $61(49-72)$ \\
\hline Diabetes, $n(\%)$ & $65(43)$ \\
\hline Coronary artery disease, $n(\%)$ & $17(11)$ \\
\hline Antihypertensive medication, $n(\%)$ & $147(98)$ \\
\hline $\mathrm{BMI}, \mathrm{kg} / \mathrm{m}^{2}$ & $27.7(24.0-30.9)$ \\
\hline Systolic blood pressure, $\mathrm{mm} \mathrm{Hg}$ & $146 \pm 16$ \\
\hline Diastolic blood pressure, $\mathrm{mm} \mathrm{Hg}$ & $79 \pm 10$ \\
\hline Pulse pressure, $\mathrm{mm} \mathrm{Hg}$ & $67 \pm 16$ \\
\hline eGFR, $\mathrm{mL} / \mathrm{min} / 1.73 \mathrm{~m}^{2}$ & $12(11-15)$ \\
\hline Creatinine, $\mu \mathrm{mol} / \mathrm{L}$ & $433(364-547)$ \\
\hline Urea, mmol/L & $20.4 \pm 4.9$ \\
\hline Hemoglobin, g/L & $114(109-121)$ \\
\hline $\mathrm{CRP}, \mathrm{mg} / \mathrm{L}$ & $3.3(1.8-6.7)$ \\
\hline Albumin, $g / L$ & $33.4 \pm 3.4$ \\
\hline Sodium, mmol/L & $141(139-142)$ \\
\hline Potassium, $\mathrm{mmol} / \mathrm{L}$ & $4.3 \pm 0.3$ \\
\hline lonized calcium, mmol/L & $1.22 \pm 0.06$ \\
\hline Phosphorus, mmol/L & $1.44(1.30-1.63)$ \\
\hline PTH, ng/L & $234(159-339)$ \\
\hline $\mathrm{pH}$ & $7.36 \pm 0.03$ \\
\hline Bicarbonate, $\mathrm{mmol} / \mathrm{L}$ & $23.3 \pm 2.3$ \\
\hline Alkaline phosphatase, U/L & $75(65-92)$ \\
\hline Total cholesterol, $\mathrm{mmol} / \mathrm{L}$ & $4.2(3.5-5.0)$ \\
\hline Low-density lipoprotein cholesterol, mmol/L & $2.3(1.6-3.0)$ \\
\hline High-density lipoprotein cholesterol, mmol/L & $1.2(1.0-1.5)$ \\
\hline Triglycerides, mmol/L & $1.5(1.1-2.0)$ \\
\hline $\mathrm{HbA} 1 \mathrm{c}, \mathrm{mmol} / \mathrm{mol}$ & $37(32-50)$ \\
\hline Ejection fraction, $n(\%)$ & $65(62-68)$ \\
\hline LVEDD, mm & $53.3 \pm 5.1$ \\
\hline LVMI, $\mathrm{g} / \mathrm{m}^{2}$ & $102(88-119)$ \\
\hline
\end{tabular}

Values are presented as mean \pm SD for normally distributed variables and median (IQR) for skewed variables. Laboratory variables are mean/median of mean values for measurements taken every 3 months from study recruitment to the control AAC imaging and echocardiographic measures are a mean of 2 consecutive assessments. Blood pressure measurements shown are a mean of all measurements during the study period. eGFR, estimated glomerular filtration rate; CRP, C-reactive protein; $\mathrm{BMI}$, body mass index; PTH, parathyroid hormone; LVEDD, left ventricular end-diastolic diameter; LVMI, left ventricular mass index; AAC, abdominal aortic calcification; SD, standard deviation; IQR, interquartile range.

surface area; left ventricular mass $=0.8\{1.04[$ [ [left ventricular enddiastolic diameter + intraventricular septum diameter + posterior wall diameter $]^{3}-$ left ventricular end-diastolic diameter $\left.\left.\left.{ }^{3}\right)\right]\right\}+0.6$. $^{2}$

\section{Statistical Analysis}

Results are presented as mean \pm standard deviation for the normally distributed variables and as median (interquartile range) for skewed variables. Normality in continuous covariates was tested with Kolmogorov-Smirnov and Shapiro-Wilk tests. Skewed vari-

Progression of Aortic Calcification in

Chronic Kidney Disease
Table 2. Medications at baseline

\begin{tabular}{ll}
\hline Medication & Patients, $n$ \\
\hline Calcium channel blockers & 122 \\
Beta-blockers & 106 \\
ACE inhibitors/angiotensin II receptor blockers & $38 / 73$ \\
Diuretics & 111 \\
Aspirin & 44 \\
Clopidogrel/ticagrelor & $8 / 1$ \\
Warfarin & 21 \\
Statins & 95 \\
Calcium-based phosphate binders & 132 \\
Calcium-based phosphate binder dose, mg & $1,000(1,000-1,000)$ \\
Non-calcium-based phosphate binders & 20
\end{tabular}

Values are presented as number of patients on medication unless stated otherwise.

ables were $\log _{\mathrm{e}}$-transformed to normalize distributions. Comparisons between AAC1 and AAC2 were done using the Wilcoxon signed-rank test. Differences between the treatment groups (conservative treatment, $\mathrm{PD}, \mathrm{HD}$, and kidney transplant) in $\triangle \mathrm{AAC}$ were examined using a nonparametric Kruskal-Wallis test, followed by the Dwass-Steel-Critchlow-Fligner method for pair-wise comparisons [14]. The mean of the 2 consecutive echocardiographic measurements was used in the analyses for echocardiographic measures, and the mean of blood pressure measurements during the study period was used for blood pressure. The mean of all consecutive laboratory measurements ( 3 months apart), respectively, was used in the analyses. Univariate and multivariable associations between exposure variables and $\triangle \mathrm{AAC}$ were examined using linear regression models. First, the associations between $\triangle \mathrm{AAC}$ and exposure variables were studied using respective univariate models. Laboratory variables were included as mean values during the interval between AAC1 and AAC2 assessments in the respective univariate models. The significant univariate exposure variables for $\triangle \mathrm{AAC}$, namely, phosphorus, albumin, LVMI, pulse pressure, and smoking status (nonsmoker, ex-smoker, and current smoker) were included in the multivariable model. Potential existence of multicollinearity was assessed by examining variance inflation factors. Associations between $\triangle \mathrm{AAC}$ and outcomes were examined using univariate Cox proportional hazards models. The low number of all-cause mortalities did not allow for multivariable models.

All statistical analyses were performed using statistical analysis system, SAS version 9.3 (SAS Institute Inc., Cary NC). A $p$ value $<0.05$ was considered statistically significant.

\section{Results}

A total of 150 patients of the CADKID study population had 2 consecutive AAC measurements (baseline: AAC1; follow-up: AAC2). The median interval between AAC measurements was 37 (29-44) months. Clinical, 


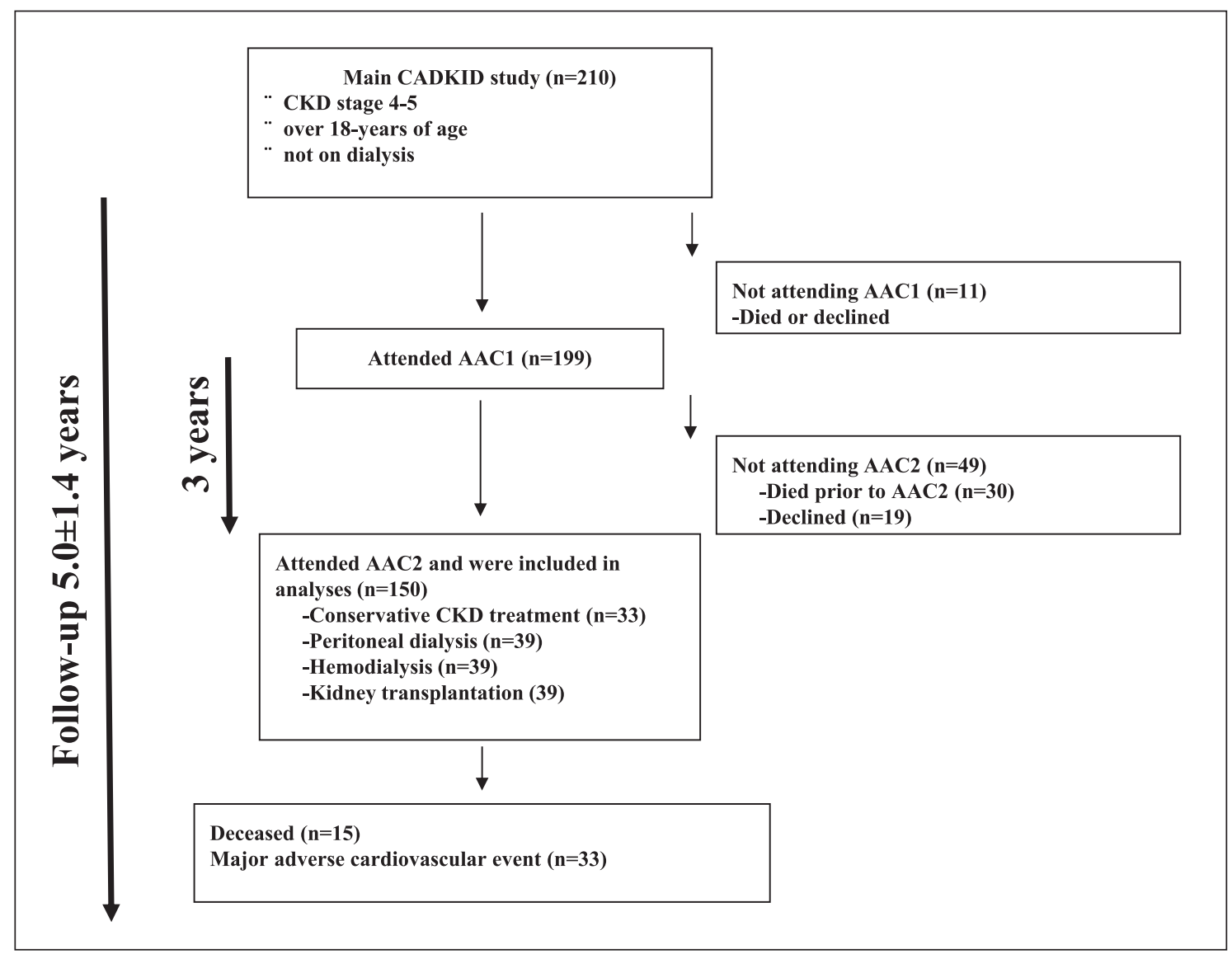

Fig. 1. Flowchart of the study. AAC1, baseline abdominal aortic calcification score; AAC2, follow-up abdominal aortic calcification; CKD, chronic kidney disease; CADKID, Chronic Arterial Disease, quality of life and mortality in chronic KIDney injury; PD, peritoneal dialysis; HD, hemodialysis; Tx, kidney transplantation; MACE, major adverse cardiovascular event.

laboratory, and echocardiographic characteristics of study subjects are shown in Table 1 and medications at baseline in Table 2.

Patients were followed up for $5.0 \pm 1.4$ years. Fifteen patients (10\%) died during the follow-up. MACEs were observed in 30 patients (22\%) during the follow-up (myocardial infarction $n=9$; stroke $n=8$; coronary artery revascularization $n=13$; no cardiovascular deaths without a prior MACE). At the time of the AAC2 assessment, 33 patients were followed up conservatively, 39 were on $\mathrm{HD}$, 39 on PD, and 39 had received a kidney transplant (Fig. 1). Only one of the patients who remained on conservative care throughout the study period was considered ineligible for RRT initiation. The eGFR at AAC2 assessment was $13 \pm 6 \mathrm{~mL} / \mathrm{min} / 1.73 \mathrm{~m}^{2}$ in the conservative treatment group. In those who started $\mathrm{HD}$ or $\mathrm{PD}$, the median time from dialysis initiation to AAC2 imaging was 19 (15-27) months, and in transplanted patients, the time from transplantation was 14 (7-22) months. In the kidney transplant recipients, eGFR was $60 \pm 23 \mathrm{~mL} / \mathrm{min} / 1.73 \mathrm{~m}^{2}$ at the time of the AAC2 imaging.

The median AAC1 was 4.8 (0.5-9.0) and median AAC2 $8.0(1.5-12.0),(p<0.0001)$. Altogether, the median $\triangle A A C$ was $0.48(0.00-1.43)$ per year. The median $\triangle \mathrm{AAC}$ was 0.41 $(0.00-0.97)$ per year in conservatively treated patients, $0.34(0.00-0.95)$ per year in transplanted patients, 0.53 $(0.00-1.57)$ per year in HD patients, and $1.0(0.0-1.83)$ per year in PD patients. The differences in $\triangle \mathrm{AAC}$ between the groups were nonsignificant ( $p=0.19$ ) (Fig. 2). Patients without calcification at baseline $(\mathrm{AAC}=0)$ had lower $\triangle \mathrm{AAC}$ than patients with any calcification at baseline $(0$ $[0-0]$ vs. $0.81[0.26-1.61]$ per year, $p<0.0001)$. There was a significant but modest correlation between AAC1 and $\triangle \mathrm{AAC}(r=0.25, p=0.002)$. The echocardiographic and blood pressure data by treatment group at baseline and at follow-up are given in online suppl. Table 1. 


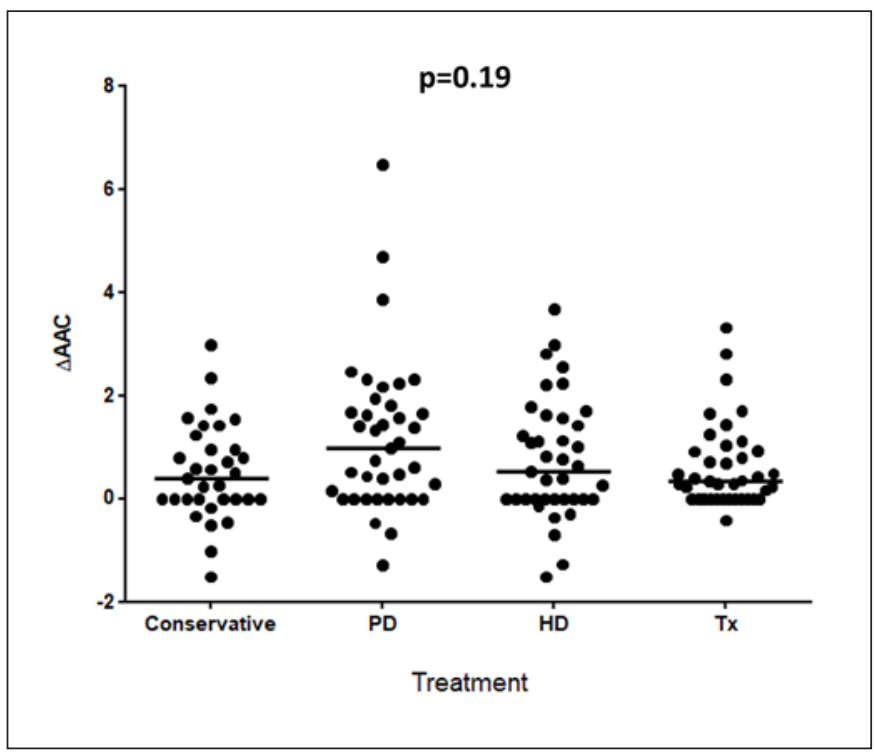

Fig. 2. Progression of $A A C$ score per 1 year $(\triangle A A C)$ in different modalities of RRT and patients continuing conservative treatment. There were no differences between the treatment groups in $\triangle \mathrm{AAC}$ $(p=0.19)$. PD, peritoneal dialysis; HD, hemodialysis; Tx, kidney transplantation; AAC, abdominal aortic calcification; RRT, renal replacement therapy.

Patients who deceased during follow-up had higher AAC1 (8.5 [5.9-15.5] vs. $4.0[0.0-8.5], p=0.004)$ and AAC2 (11.5 [8.5-18.0] vs. $7.0[1.0-11.5], p=0.003)$ and higher $\triangle \mathrm{AAC}[1.67(0.0-2.25]$ vs. $0.43[0.00-1.27], p=$ 0.04 ) than others. $\triangle \mathrm{AAC}$ (HR 1.427, 95\% confidence interval [CI] 1.044-1.950, $p=0.03$ ), AAC1 (HR 1.128, 95\% CI 1.043-1.220, $p=0.003$ ), and AAC2 (HR 1.140, 95\% CI $1.050-1.237, p=0.002)$ were associated with all-cause mortality in univariate Cox proportional hazards models. AAC1 (HR 1.065 95\% CI 1.003-1.130, $p=0.04$ ) and AAC2 (HR $1.06795 \%$ CI 1.010-1.128, $p=0.02$ ) were associated with incident MACEs but $\triangle \mathrm{AAC}$ was not (HR 1.194 95\% CI 0.921-1.549, $p=0.18$ ).

In univariate analysis, $\triangle \mathrm{AAC}$ was significantly associated with mean phosphorus (log phosphorus: $\beta=1.79$, $p=0.001)$, mean albumin $(\beta=-0.09, p=0.0005)$, mean LVMI (log LVMI: $\beta=1.43, p=0.0005)$, mean pulse pressure $(\beta=0.02, p=0.009)$, and smoking status (nonsmoker, ex-smoker, and current smoker: $\beta=0.31, p=0.02$ ). Serum lipids, glycated hemoglobin, ionized or total calcium, PTH, or other repeated laboratory variables were not significantly associated with $\triangle \mathrm{AAC}$. In the multivariable model, the only significant explanatory variables for $\triangle \mathrm{AAC}$ were mean LVMI (log LVMI: $\beta=0.97, p=0.02)$ and phosphorus (log phosphorus: $\beta=1.19, p=0.02)(\mathrm{Ta}-$
Table 3. Univariate and multivariable associations between risk factors and $\triangle \mathrm{AAC}$

\begin{tabular}{|c|c|c|c|c|}
\hline \multirow[t]{2}{*}{ Variable } & \multicolumn{2}{|c|}{$\begin{array}{l}\text { Univariate } \\
\text { analysis }\end{array}$} & \multicolumn{2}{|c|}{$\begin{array}{l}\text { Multivariate } \\
\text { analysis }\end{array}$} \\
\hline & $\beta$ & $p$ value & $\beta$ & $p$ value \\
\hline Mean phosphorus, log & 1.79 & 0.001 & 1.19 & 0.02 \\
\hline Mean albumin, $\mathrm{g} / \mathrm{L}$ & -0.09 & 0.0005 & -0.04 & 0.17 \\
\hline Mean LVMI, log & 1.43 & 0.0005 & 0.97 & 0.02 \\
\hline Smoking status & 0.31 & 0.02 & 0.19 & 0.16 \\
\hline Mean pulse pressure, $\mathrm{mm} \mathrm{Hg}$ & 0.02 & 0.009 & 0.005 & 0.41 \\
\hline
\end{tabular}

log, logarithmic transformation; smoking status (nonsmoker, exsmoker, and current smoker). LVMI, left ventricular mass index; AAC, abdominal aortic calcification.

ble 3). The progression of LVMI was however not associated with $\triangle \mathrm{AAC}$ (data not shown).

The use of calcium-based phosphate binders or their dose was not associated with $\triangle \mathrm{AAC}$ nor was the use of non-calcium-based phosphate binders. Moreover, warfarin use was not associated with $\triangle \mathrm{AAC}$.

In those who had received a transplant, the time to transplantation from the beginning of the study was associated with $\triangle \mathrm{AAC}$ (per month on the waiting list: $\beta=$ $0.04, p=0.001)$. However, the time from transplantation to the AAC2 imaging was not associated with $\triangle \mathrm{AAC}(\beta=$ $-0,000, p=0.53)$, and in dialysis patients, the duration of dialysis therapy was not associated with $\triangle \mathrm{AAC}(\beta=0.009$, $p=0.48)$.

\section{Discussion}

The current study shows for the first time that AAC progression is similar in CKD stage 4-5 patients transitioning to different modalities of RRT or continuing conservative treatment during a median 3-year follow-up. The increase rate of AAC was independently associated with plasma phosphorus but not ionized calcium, $\mathrm{PTH}$, alkaline phosphatase, serum lipids, glycated hemoglobin, or other laboratory variables examined every 3 months from the beginning of the study to the latter AAC assessment.

Several previous studies have assessed the progression of $\mathrm{VC}$ in patients with various stages of CKD and shown that calcification progresses rapidly in earlier stages of $\mathrm{CKD}$, patients on maintenance dialysis, and even kidney transplant patients with functional grafts $[10,11,15]$. 
However, until now, comparative data have been lacking on patients assessed for AAC during advanced CKD prior to RRT initiation and prospectively after transitioning to different treatment modalities or continuing conservative treatment. Kidney transplantation is indisputably the best way to decrease mortality risk in CKD stage 5 patients. Therefore, it is surprising that patients receiving a transplant prior to the second AAC assessment had similar AAC progression compared to the other groups. Our current findings are however in line with previous studies demonstrating that $26-64 \%$ of transplant patients show progression of AAC in a mean follow-up of 1-3 years [9, 15]. Although $\triangle \mathrm{AAC}$ was positively associated with the time on the transplant waiting list in the present study, the time from transplantation to the control AAC imaging was not associated with $\triangle \mathrm{AAC}$. These findings suggest that at least in the early years following kidney transplantation, the VC process continues similarly to that on maintenance dialysis or without RRT altogether. The median time from transplantation to the second AAC assessment was however rather short in the present study (14 months).

Patients who deceased during follow-up had higher $\mathrm{AAC} 1, \mathrm{AAC} 2$, and $\triangle \mathrm{AAC}$ than others, and $\triangle \mathrm{AAC}$ was associated with mortality in a univariate Cox proportional hazards model. However, the low number of deaths did not allow for multivariable models. AAC1 and AAC2 were associated with incident MACEs but $\triangle \mathrm{AAC}$ was not. This may suggest that the presence of calcification in advanced CKD is indicative of future cardiovascular events and mortality even when it is not progressing further. In line with this assumption, Inoue et al. [16] recently demonstrated that although a trajectory of longitudinal high or rapid VC was associated with increased risk of death among 396 chronic HD patients, during a mean followup of 2 years, the progression of calcification was not predictive of mortality in patients without baseline VC. In the present study, patients with positive AAC at baseline had higher $\triangle \mathrm{AAC}$ than those who were free of calcification in the beginning of the study.

Cardiovascular disease is the leading cause of death in patients with advanced CKD, and attempts to improve prognosis should be focused on its treatment and prevention [1]. Traditional risk factors explain only a part of the increased risk, and nonclassical kidney-specific variables contribute significantly. Cardiovascular risk is diminished by kidney transplantation, but nevertheless, cardiovascular sequelae remain the leading cause of death after transplantation $[17,18]$. CKD-MBD syndrome has been implicated as a significant risk factor for arterial calcifica- tion, MACEs, and mortality in CKD. Previous studies in advanced CKD including patients with or without maintenance dialysis and kidney transplant recipients have shown that increased calcium, phosphorus, and PTH levels are associated with accelerated progression of aortic calcification $[9-11,15]$. In the present study, plasma phosphorus was the only repeated laboratory variable independently associated with $\triangle \mathrm{AAC}$. Other laboratory variables associated with CKD-MBD such as $\mathrm{PTH}$, total or ionized calcium, or alkaline phosphatase were not significantly associated with $\triangle \mathrm{AAC}$. Moreover, serum lipids, glycated hemoglobin, or C-reactive protein were not associated with $\triangle \mathrm{AAC}$, and the association between plasma albumin and $\triangle \mathrm{AAC}$ became insignificant after adjusting for other explanatory variables in the multivariable model.

Although phosphorus levels even in the normal range have been established as an undisputed risk factor for cardiovascular and all-cause mortality in CKD by epidemiological studies [19], the few available randomized studies on the effects of phosphate-lowering interventions on VC have been disappointing. The potential benefits of phosphate-lowering therapy in patients with predialytic CKD remain to be demonstrated. However, most studies in CKD patients without maintenance dialysis have included patients with normal or only slightly elevated phosphorus levels at baseline. The reduction in phosphate observed with lanthanum carbonate, sevelamer, or calciumbased phosphatebindershasbeenstatisticallyinsignificant, and no improvement in surrogate markers for arteriosclerosis or VC has been observed [20-22]. Conversely, in maintenance dialysis patients, recent meta-analyses have demonstrated a benefit for non-calcium-based phosphate binders for managing hyperphosphatemia with a parallel reduction in VC and mortality [23, 24]. Therefore, patients with apparent baseline VC and advanced CKD soon transitioning to RRT may benefit from assessment of CKD-MBD and management of hyperphosphatemia to limit the progression of VC.

Left ventricular hypertrophy has been associated with extra-coronary atherosclerosis and VC in maintenance HD patients [25], and the prevalence of left ventricular hypertrophy is high in CKD. Arteriosclerosis increases the stiffness of the aorta and large capacitance arteries, leading to left ventricular hypertrophy via pressure overload. In the present study however, the progression of LVMI during the study period was not associated with $\triangle \mathrm{AAC}$, although mean LVMI was.

The current study has limitations. First, the study was conducted in a single center, and the sample size was
Lankinen/Hakamäki/Hellman/Koivuviita/ Metsärinne/Järvisalo 
somewhat limited. Assessing aortic calcification on the basis of plain radiographs may overlook some elusive calcifications and changes compared with CT techniques. However, intra- and interobserver reproducibility of the AAC measurements were acceptable. In comparison to previous studies, our study setting made it possible to compare AAC progression between different modalities of RRT and led to the novel finding that AAC progression during the early years following kidney transplantation is similar to that observed in patients initiating maintenance dialysis or continuing on conservative treatment. As the primary aim of the study was to examine the development of $\mathrm{AAC}$ and associated risk factors in $\mathrm{CKD}$ stage 4-5 patients transitioning to RRT or continuing on conservative care, we only included patients with 2 consecutive AAC assessments. As several patients deceased prior to the AAC2 assessment, the association between $\triangle \mathrm{AAC}$ and adverse events is likely to be affected by the study design and should be interpreted with caution. The assessment of laboratory parameters' associations with $\triangle \mathrm{AAC}$ was based on a mean of several measurements performed every 3 months spanning the interval from study recruitment to the latter AAC imaging, increasing the statistical weight and reliability of our current findings.

In conclusion, our current data show for the first time that AAC progresses at a comparable rate in patients on different RRT modalities and patients continuing conservative care during a follow-up of 3 years. Furthermore, AAC is associated with mortality and MACEs. Plasma phosphorus and LVMI are independently associated with $\triangle \mathrm{AAC}$.

\section{Statement of Ethics}

The study protocol (reference No. T05/024/20) was approved by the Turku University Clinical Research Center and Turku University Hospital Scientific Ethics Review Board and the Hospital district of Southwest Finland. All procedures were in accordance with the Helsinki Declaration. All patients gave written informed consent before entering the study.

\section{Conflict of Interest Statement}

The authors have no conflicts of interest to declare.

\section{Funding Sources}

This work was supported by research grants from Finska Läkaresällskapet and Perklén Foundation, Helsinki, Finland. The grants were used to collect and analyze the data. The funding bodies were not involved with the design of the study or interpretation of the data.

\section{Author Contributions}

R.L., M.J.J., M.H., N.S.K., T.H., and K.M. designed the study and were responsible for the data collection. M.J.J. performed the statistical analyses. R.L. and M.J.J. drafted the manuscript. M.H., N.S.K., T.H., and K.M. revised the manuscript.

\section{Data Availability Statement}

Data that support the findings of the current study are available from the data sets of the Department of Nephrology and the Informatics Department of Turku University Hospital on reasonable request from the corresponding author and after permission of the Ethics Committee of Turku University Hospital.

\section{References}

1 Foley RN, Murray AM, Li S, Herzog CA, McBean AM, Eggers PW, et al. Chronic kidney disease and the risk for cardiovascular disease, renal replacement, and death in the United States medicare population, 1998 to 1999. J Am Soc Nephrol. 2005 Feb;16(2):489-95.

2 Pencak P, Czerwieńska B, Ficek R, Wyskida K, Kujawa-Szewieczek A, OlszaneckaGlinianowicz M, et al. Calcification of coronary arteries and abdominal aorta in relation to traditional and novel risk factors of atherosclerosis in hemodialysis patients. BMC Nephrol. 2013 Jan;14:10.

3 KDIGO Work Group. Clinical practice guideline for the evaluation and management of chronic kidney disease. Kidney Int Suppl. 2013 Jan;3(Suppl 3):1-150.

4 Davies MR, Hruska KA. Pathophysiological mechanisms of vascular calcification in end- stage renal disease. Kidney Int. 2001 Aug; 60(2):472-9.

5 Peeters MJ, van den Brand JA, van Zuilen AD, Koster Y, Bots ML, Vervloet MG, et al. Abdominal aortic calcification in patients with CKD. J Nephrol. 2017 Feb;30(1):109-18.

6 Lewis JR, Schousboe JT, Lim WH, Wong G, Wilson KE, Zhu K, et al. Long-term atherosclerotic vascular disease risk and prognosis in elderly women with abdominal aortic calcification on lateral spine images captured during bone density testing: a prospective study. J Bone Miner Res. 2018 Jun;33(6): 1001-10.

7 Niu Q, Hong Y, Lee CH, Men C, Zhao H, Zuo L. Abdominal aortic calcification can predict all-cause mortality and CV events in dialysis patients: a systematic review and meta-analysis. PLoS One. 2018 Sep;13(9):e0204526.
8 Imanishi K, Hatakeyama S, Yamamoto $\mathrm{H}$ Okamoto A, Imai A, Yoneyama T, et al. Posttransplant renal function and cardiovascular events are closely associated with the aortic calcification index in renal transplant recipients. Transpl Proc. 2014;46(2):484-8.

9 Meneghini M, Regalia A, Alfieri C, Barretta F, Croci D, Gandolfo MT, et al. Calcium and osteoprotegerin levels predict the progression of the abdominal aortic calcifications after kidney transplantation. Transplantation. 2013 Jul;96(1):42-8.

10 Noordzij M, Cranenburg EM, Engelsman LF, Hermans MM, Boeschoten EW, Brandenburg VM, et al. Progression of aortic calcification is associated with disorders of mineral metabolism and mortality in chronic dialysis patients. Nephrol Dial Transplant. 2011 May; 26(5):1662-9. 
11 Yamamoto D, Suzuki S, Ishii H, Hirayama K, Harada K, Aoki T, et al. Predictors of abdominal aortic calcification progression in patients with chronic kidney disease without hemodialysis. Atherosclerosis. 2016 Oct;253: $15-21$.

12 Lankinen R, Hakamäki M, Metsärinne K, Koivuviita NS, Pärkkä JP, Hellman T, et al. Cardiovascular determinants of mortality in advanced chronic kidney disease. Am J Nephrol. 2020;51(9):726-35.

13 Kauppila LI, Polak JF, Cupples LA, Hannan MT, Kiel DP, Wilson PW. New indices to classify location, severity and progression of calcific lesions in the abdominal aorta: a 25year follow-up study. Atherosclerosis. 1997 Jul;132(2):245-50.

14 Critchlow DE, Fligner MA. On distributionfree multiple comparisons in the one-way analysis of variance. Commun Stat Theor Methods. 1991;20:127-39.

15 Naganuma T, Takemoto Y, Uchida J, Nakatani T, Kabata D, Shintani A. Hypercalcemia is a risk factor for the progression of aortic calcification in kidney transplant recipients. Kidney Blood Press Res. 2019; 44(4):823-34.
16 Inoue $\mathrm{H}$, Shimizu S, Watanabe K, Kamiyama $\mathrm{Y}$, Shima $\mathrm{H}$, Nakase A, et al. Impact of trajectories of abdominal aortic calcification over 2 years on subsequent mortality: a 10-year longitudinal study. Nephrol Dial Transplant. 2018 Apr;33(4):676-83.

17 Kahwaji J, Bunnapradist S, Hsu JW, Idroos ML, Dudek R. Cause of death with graft function among renal transplant recipients in an integrated healthcare system. Transplantation. 2011 Jan;91(2):225-30.

18 Ojo AO, Morales JM, González-Molina M, Steffick DE, Luan FL, Merion RM, et al. Comparison of the long-term outcomes of kidney transplantation: USA versus Spain. Nephrol Dial Transplant. 2013 Jan;28(1):213-20.

19 Block GA, Klassen PS, Lazarus JM, Ofsthun N, Lowrie EG, Chertow GM. Mineral metabolism, mortality, and morbidity in maintenance hemodialysis. J Am Soc Nephrol. 2004 Aug;15(8):2208-18.

20 Toussaint ND, Pedagogos E, Lioufas NM, Elder GJ, Pascoe EM, Badve SV, et al. A randomized trial on the effect of phosphate reduction on vascular end points in CKD (IMPROVE-CKD). J Am Soc Nephrol. 2020 Nov; 31(11):2653-66.
21 Kovesdy CP, Lu JL, Wall BM, Gyamlani G, Naseer A, Wallick A, et al. Changes with lanthanum carbonate, calcium acetate, and phosphorus restriction in CKD: a randomized controlled trial. Kidney Int Rep. 2018 Mar;3(4):897-904.

22 Chue CD, Townend JN, Moody WE, Zehnder D, Wall NA, Harper L, et al. Cardiovascular effects of sevelamer in stage 3 CKD. J Am Soc Nephrol. 2013 Apr;24(5):842-52.

23 Patel L, Bernard LM, Elder GJ. Sevelamer versus calcium-based binders for treatment of hyperphosphatemia in CKD: a meta-analysis of randomized controlled trials. Clin J Am Soc Nephrol. 2016 Feb;11(2):232-44.

24 Liu L, Wang Y, Chen H, Zhu X, Zhou L, Yang $Y$. The effects of non-calcium-based phosphate binders versus calcium-based phosphate binders on cardiovascular calcification and bone remodeling among dialysis patients: a meta-analysis of randomized trials. Ren Fail. 2014 Sep;36(8):1244-52.

25 Yildiz A, Memisoglu E, Oflaz $\mathrm{H}$, Yazici $\mathrm{H}, \mathrm{Pu}$ suroglu $\mathrm{H}$, Akkaya V, et al. Atherosclerosis and vascular calcification are independent predictors of left ventricular hypertrophy in chronic haemodialysis patients. Nephrol Dial Transplant. 2005 Apr;20(4):760-7. 